NOTA

\title{
Determinación de mercurio en muestras biológicas prehispánicas colombianas: primeras experiencias y perspectivas de investigación
}

\author{
Alvaro J. Idrovo ${ }^{1}$, William M. Romero ${ }^{2}$, Elizabeth Silva ${ }^{3}$, Gladys Villamil de García ${ }^{3}$, Jaime E. Ortiz ${ }^{3}$ \\ ${ }^{1}$ Departamento de Salud Pública y Tropical, Facultad de Medicina, Universidad Nacional de Colombia, \\ Bogotá, D.C., Colombia. \\ ${ }^{2}$ Departamento de Antropología, Facultad de Ciencias Humanas, Universidad Nacional de Colombia, \\ Bogotá, D.C., Colombia. \\ ${ }^{3}$ Laboratorio de Salud Ambiental, Instituto Nacional de Salud, Bogotá, D.C., Colombia.
}

\begin{abstract}
El mercurio puede servir de trazador del grado de contaminación ambiental. En este trabajo se midió el mercurio mediante técnicas convencionales en el cabello de dos momias de los siglos XII y XIV, una adulta y otra infantil, y en el pelo de un venado (Odocoileus virginianus). Se encontraron muy bajos niveles de mercurio total e inorgánico, probablemente debido a la mínima exposición alimentaria y a la ausencia de contaminación atmosférica. Es posible utilizar estas técnicas para explorar la relación hombre-ambiente, especialmente después de la aparición de la metalurgia, y para estimar la contaminación ambiental en diferentes períodos.
\end{abstract}

Palabras clave: paleopatología, mercurio, contaminación ambiental, toxicología arqueológica.

\section{Determination of mercury in Colombian prehispanic biological samples}

Mercury is useful as a tracer of environmental pollution levels. We measured mercury levels in hair from two human mummies (XII and XIV centuries, respectively) and from a stag (Odocoileus virginianus). The total and inorganic mercury levels found in the samples were very low. This findings indicated a minimal exposure to mercury in food and its absence in the atmosphere. Mercury levels can be used to explore the relationship between humans and environment, especially after metallurgy appeared, and to assess environmental contamination in different periods.

Key words: paleopathology, mercury, environmental pollution, archaeological toxicology.

La paleopatología estudia la enfermedad en comunidades primitivas (1), principalmente mediante el diagnóstico anatomopatológico de los restos humanos (2). En Colombia, las enfermedades más estudiadas son la tuberculosis, la sífilis, el pian y la leishmaniasis (3-8), debido a la importancia de las enfermedades infecciosas en períodos primitivos (9). Este enfoque está basado en la enfermedad y no en los factores que se pueden asociar con su ocurrencia. Las enfermedades no infecciosas que se asocian con

Correspondencia:

Alvaro J. Idrovo

idrovoaj@hotmail.com.

Recibido: 25/09/01; aceptado: 08/02//02 agentes químicos han aumentado su ocurrencia con el paso del tiempo, especialmente desde que los procesos de urbanización e industrialización fueron más generalizados (10). Una forma de explorar estos cambios en la morbimortalidad es mediante el uso de biomarcadores no perecederos después de la muerte del individuo, lo que se ha denominado toxicología arqueológica (11).

En la actualidad hay pocos estudios que exploren la exposición a agentes químicos y la ocurrencia de enfermedades en individuos procedentes de períodos lejanos en el tiempo. En el hueso se ha estudiado plomo, cadmio, cinc, plata, arsénico, flúor y mercurio (12-24). El cabello ha sido utilizado para cuantificar benzoilecognina, metabolito de la coca, selenio, mercurio y cadmio $(25,26)$. El 
mercurio, que no es esencial para los seres vivos (27), se puede determinar en forma inorgánica y orgánica en el cabello. La primera forma es relativamente insoluble, generalmente no representa una amenaza para la cadena alimenticia humana y su importancia radica básicamente en la exposición inhalatoria. La forma orgánica puede ingresar al organismo por vía digestiva (28), razón por la cual el mercurio orgánico es un buen indicador de la exposición a través del alimento.

El presente trabajo piloto tuvo como objetivo determinar las concentraciones de mercurio en tres muestras biológicas prehispánicas de Colombia mediante técnicas modificadas y validadas en el Laboratorio de Salud Ambiental del Instituto Nacional de Salud y revisar su utilidad en la paleopatología.

Se midió el mercurio total e inorgánico en tres muestras biológicas prehispánicas procedentes de La Mesa de los Santos (Santander), que se encuentran asociadas arqueológicamente a la cultura Guane (siglos VIII-XII d.C.). Las dos primeras muestras se encuentran en el Museo Arqueológico Casa del Marqués de San Jorge (29) y la tercera en el Instituto de Ciencias Naturales de la Universidad Nacional de Colombia.

La primera muestra corresponde a un individuo femenino de entre 3 y 5 años, de acuerdo con la secuencia de formación y erupción dental (30), en buen estado de conservación (figura 1). Sus características más relevantes son: 1) posición fetal, 2) envuelta en fardos (muestra 2), 3) tipo de momificación artificial, y 4) sin evisceración. El cabello es de color café rojizo de aproximadamente $25 \mathrm{~cm}$ de largo, con extremos distales con horquilla y huevecillos de piojos.

La segunda muestra es una piel de venado (Odocoileus virginianus) utilizada como fardo funerario de la muestra 1, lo cual era frecuente entre las culturas de filiación Chibcha. Este animal era común en esta zona (31).

La tercera muestra es un cráneo parcialmente momificado de un adulto masculino, de entre 30 y 35 años, con abundante cabello negro de 25 a 30 $\mathrm{cm}$ de largo. No tiene un amplio diagnóstico antropológico.

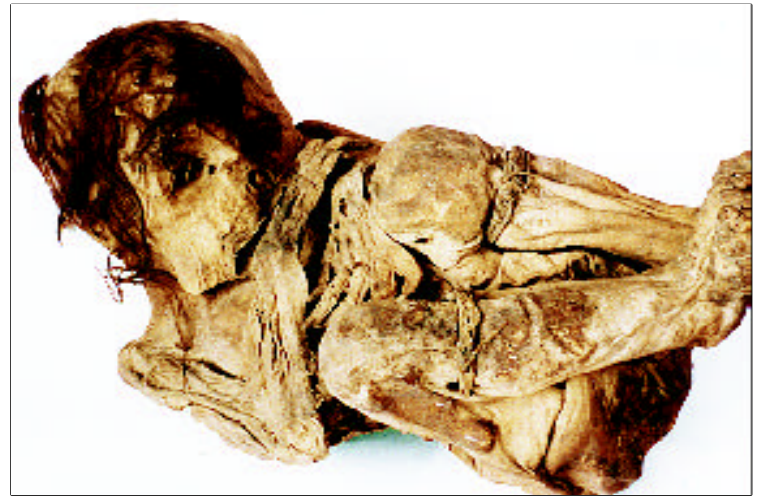

Figura 1. Restos momificados correspondientes a la infante de la muestra 1.

La muestra 2 se obtuvo por rasurado del material, y las muestras 1 y 3 mediante corte del cabello. Las técnicas de lavado, digestión y análisis químico de las muestras se encuentran detalladas extensamente en otro documento (32). Las concentraciones detectadas se encuentran resumidas en el cuadro 1. Llama la atención la presencia de mercurio inorgánico a nivel de trazas en la muestra 2, si se tiene en cuenta que estas poblaciones no tenían actividades que generaran exposiciones a este metal y la concentración esperada era indetectable $(<0,3 \mu \mathrm{g} / \mathrm{g})$.

En Colombia se han descrito niveles de hasta 770 $\mu \mathrm{g} / \mathrm{g}$ en individuos expuestos en la minería aurífera (33), siendo los valores promedio y mediana de $8,73 \mu \mathrm{g} / \mathrm{g}$ y $1,8 \mu \mathrm{g} / \mathrm{g}$, respectivamente (datos del Laboratorio de Salud Ambiental, INS). Por tanto, se puede sugerir que la contaminación por mercurio ha aumentado con el paso del tiempo. Su presencia se puede relacionar con el grado de contaminación ambiental o con algunas actividades propias del individuo y la sociedad a la cual perteneció. Esto es especialmente cierto en los últimos dos siglos, ya que existen evidencias que muestran que los niveles actuales de mercurio en la atmósfera son tres veces mayores que los de hace 150 años (34).

Las concentraciones halladas pueden tener como origen fuentes naturales y antropogénicas. El mercurio inorgánico pudo relacionarse con actividades cotidianas como la combustión de carbón, madera y malezas, y a fenómenos 
Cuadro 1. Concentraciones de mercurio en las muestras biológicas prehispánicas estudiadas.

\begin{tabular}{lccc}
\hline & \multicolumn{3}{c}{ Concentración $(\boldsymbol{\mu g} / \mathbf{g})$} \\
\cline { 2 - 4 } Muestra & Total & Inorgánico & Orgánico \\
\hline Pelo Odocoileus virginianus (Fardo) & 0,7 & $<0,3^{*}$ & 0,6 \\
Cabello individuo infantil (total) & $<0,3$ & $<0,3$ & $<0,3$ \\
Cabello individuo adulto & & $<0,3$ & $<0,3$ \\
Proximal & $<0,3$ & $<0,3$ & -- \\
Distal & $<0,3$ & & \\
\hline
\end{tabular}

${ }^{*}$ Valor alrededor de $0,1 \mu \mathrm{g} / \mathrm{g}$.

naturales como erupciones volcánicas, incendios forestales, evaporación por procesos biológicos y erosión de suelos con alto contenido del metal. El mercurio orgánico podría reflejar su presencia en el agua y principalmente en la alimentación (35). Los niveles de mercurio aumentan a medida que se avanza en la escala de los predadores; por ello, una dieta rica en tejido animal tiene mayor contenido de mercurio que una dieta basada en alimentos vegetales o lactovegetariana $(36,37)$.

Egeland y colaboradores (26) determinaron en un estudio el contenido de mercurio total, metilmercurio, selenio y cadmio en 16 muestras procedentes de un sitio arqueológico en Karluk (Alaska), correspondiente a culturas con 400 a 800 años de antigüedad. Los niveles de metil-mercurio fueron de $0,03 \mathrm{ppm}(\mathrm{DE}=0,02)$, que corresponde aproximadamente al $2 \%$ (1,5 ppm) de los niveles de mercurio total encontrado. Estos resultados son similares a los descritos en esta publicación, lo cual refuerza la evidencia del incremento de contaminación ambiental debida al desarrollo industrial.

La experiencia descrita muestra la factibilidad de realizar estudios toxicológicos en muestras biológicas antiguas bien conservadas. Mediante esta metodología, se pueden hacer aproximaciones con respecto al grado de exposición a mercurio y a otros metales contaminantes entre individuos prehispánicos y, en general, de épocas pasadas. De especial importancia son las épocas en las cuales se introdujo la metalurgia y, posteriormente, el inicio de la industrialización. Futuras investigaciones que utilicen las técnicas aquí descritas o similares podrán contribuir a determinar los niveles basales de contaminación en una región determinada, el comportamiento de la contaminación ambiental y los potenciales efectos tóxicos en las poblaciones (11).

\section{Referencias}

1. Campillo D. La enfermedad en la prehistoria. Introducción a la paleopatología. Barcelona: Salvat; 1983. p.1-10.

2. Roberts C, Manchester K. The archaeology of disease. Second edition. Cornell: Cornell University Press; 1997.

3. Correal-Urrego G. Algunas enfermedades precolombinas. Rev Univ Nal 1985;1:14-27.

4. Idrovo AJ. Tuberculosis prehispánica en Muiscas de la Sabana de Bogotá. Rev Fac Med UN Col 1997;45:50-4.

5. Rodríguez JV, Etxeberria F. La tuberculosis en la Colombia prehispánica. Bol Asoc Esp Paleopatol 1998; 19:8-17.

6. Rodríguez JV, Rodríguez CA, Bernal F. Dos posibles casos de treponematosis en restos prehispánicos del Valle del Cauca, Colombia. Maguaré Rev Departamento Antropol UN Colombia 1998;13:85-98.

7. Romero WM. Mal de Pott en momia de la colección del museo arqueológico Marqués de San Jorge. Maguaré Rev Departamento Antropol UN Col 1998;13:99-115.

8. Sotomayor HA. Arqueomedicina de Colombia prehispánica. Santafé de Bogotá: Taller Editorial Cafam; 1992. p.1-80.

9. McKeown T. Los orígenes de las enfermedades humanas. Barcelona: Editorial Crítica; 1990. p.1-316.

10. Frenk J, Bobadilla JL, Stern C, Lozano R, Sepúlveda J, José M. La transición epidemiológica en América Latina. Bol Of Sanit Panam 1991;111:485-96.

11. Ericson JE, Coughlin EA. Archaeological toxicology. Ann NY Acad Sci 1981;376:393-403.

12. Barry PS, Connolly R. Lead concentrations in mediaeval bones. Int Arch Occup Enviorn Health 1981;48:173-7.

13. Ericson JE, Shirahata H, Patterson CC. Skeletal concentrations of lead in ancient Peruvians. N Engl J Med 1979;300:946-51. 
14. Ericson JE, Smith DR, Flegal AR. Skeletal concentrations of lead, cadmium, zinc, and silver in ancient North American Pecos Indians. Environ Health Perspect 1991;93:217-23.

15. Harper M. Possible toxic metal exposure of prehistoric bronze workers. Br J Ind Med 1987;44:652-6.

16. Hisanaga A, Hirata M, Tanaka A, Ishinishi N, Eguchi Y. Variation of trace metals in ancient and contemporary Japanese bones. Biol Trace Elem Res 1989;22:221-31.

17. Jaworowski Z, Barbalat F, Blain C, Peyre E. Heavy metals in human and animal bones from ancient and contemporary France. Sci Total Environ 1985;43:10326.

18. Klys M, Lech T, Zi eba-Palus J, Bialka Jendereço. A chemical and physicochemical study of an Egyptian mummy 'Iset Iri Hetes' from the Ptolemaic period III-I B.C. Forensic Sci Int 1999;99:217-28.

19. Patterson C, Ericson J, Manea-Krichten M, Shirahata H. Natural skeletal levels of lead in Homo sapiens sapiens uncontaminated by technological lead. Sci Total Environ 1991;107:205-36.

20. Patterson CC, Shirahata H, Ericson JE. Lead in ancient human bones and its relevance to historical developments of social problems with lead. Sci Total Environ 1987;61:167-200.

21. Saltzman BE, Gross SB, Yeager DW, Meiner BG, Gartside PS. Total body burdens and tissue concentrations of lead, cadmium, copper, zinc, and ash in 55 human cadavers. Environ Res 1990;52:126-45.

22. Settle DM, Patterson CC. Lead in albacore: guide to lead pollution in Americans. Science 1980;207:1167-76.

23. Soriano $\mathbf{M}$. The fluoric origin of the bone lesion in the Pithecanthropus erectus femur. Am J Phys Anthropol 1970;32:49-57.

24. Waldron HA. Lead poisoning in the ancient world. Med Hist 1973;17:391-9.

25. Cartmell LW, Aufderhide A, Weems C. Cocaine metabolites in pre-Columbian mummy hair. J Okla State Med Assoc 1991;84:11-2.

26. Egeland GMAU, Ponce RAU, Knecht RAU, Bloom NSAU, Fair JAU, Middaugh JP. Trace metals in ancient hair from the Karluk Archaeological Site, Kodiak, Alaska. Int J Circumpolar Health 1999;58:52-6.

27. Markert B. Presence and significance of naturally occurring chemical elements of the periodic system in the plant organism and consequences for future investigations on inorganic environmental chemistry in ecosystems. Vegetatio 1992;103:1-30.

28. Mailman RB. Heavy metals. In: Guthrie FE, Perry JJ, editors. Introduction to environmental toxicology. New York: Elsevier; 1980. p.34-43.

29. Romero WM. Estudio bioantropológico de las momias del Museo Arqueológico Marques de San Jorge (tesis). Santafé de Bogotá: Universidad Nacional de Colombia; 1997.

30. Ubelaker D. Human skeletal remains. Washington: Smithsonian Institution; 1989. p.90.

31. Simón P. Noticias historiales de las conquistas de tierra firme en las Indias Occidentales.Bogotá: Biblioteca Banco Popular; 1981.

32. Podlesky EO, Ortiz JE, de García GV. Determinación de trazas de metales en muestras biológicas y ambientales. Santafé de Bogotá: Instituto Nacional de Salud; 1992.

33. Idrovo AJ, Manotas LE, Villamil de García G, Ortiz JE, Silva E, Romero SA, et al. Niveles de mercurio y percepción del riesgo en una población minera aurífera del Guainía (Orinoquia colombiana). Biomédica 2001;21:134-41.

34. Swain EB, Engstrom DR, Brigham ME, Henning TA, Brezonik PL. Increasing rates of atmospheric mercury deposition in midcontinental North America. Science 1992; 257:784-7.

35. Morel FMM, Kraepiel AML, Amyot M. The chemical cycle and bioaccumulation of mercury. Annu Rev Ecol Syst 1998;29:543-66.

36. Airey D. Mercury in human hair due to environment and diet: a review. Environ Health Perspect 1983;52:303-16.

37. Srikumar TS, Johansson GK, Ockerman PA, Gustatsson JA, Akesson B. Trace elements status in healthy subjects switching from a mixed to a lactovegetarian diet for 12 mo. Am J Clin Nutr 1992;55: 885-90. 